\title{
summary
}

\section{Paracetamol an effective analgesic, but adding codeine gives additional benefit}

Moore A, Collins S, Carroll D, McQuay HJ. Paracetamol with and without codeine in acute pain: a quantitative systematic review. Pain 1997; 77:193-201

Objective To assess the analgesia obtained from single oral doses of paracetamol alone and with codeine.

Data sources A search of the following databases, Medline 19661996, Embase 1980-1996, Cochrane Library Issue 2, 1996, Oxford Pain Relief Database 1950-1994, reference lists and textbooks, using a detailed search strategy.

Study selection Only full journal publication of double-blind studies with randomly allocated adult patients receiving postoperative oral administration for treatment of moderate to severe pain baseline pain (equates to $>30 \mathrm{~mm}$ on a visual analogue scale, VAS), using acceptable pain measures.

Results Thirty-one trials met the inclusion criteria of which 19 related to dental pain for paracetamol versus placebo, 19 trials for paracetamol and codeine versus placebo (14 dental) and 13 for paracetamol and codeine versus paracetamol alone (10 dental).

Conclusion Paracetamol is an effective analgesic, and the addition of $60 \mathrm{mg}$ of codeine produces worthwhile additional pain relief.

Address for reprints: Dr A Moore, Pain Research and Nuffield Department of Anaesthetics, University of Oxford, Oxford Radcliffe NHS Trust, The Churchill, Headington, Oxford OX3 7LJ, UK.

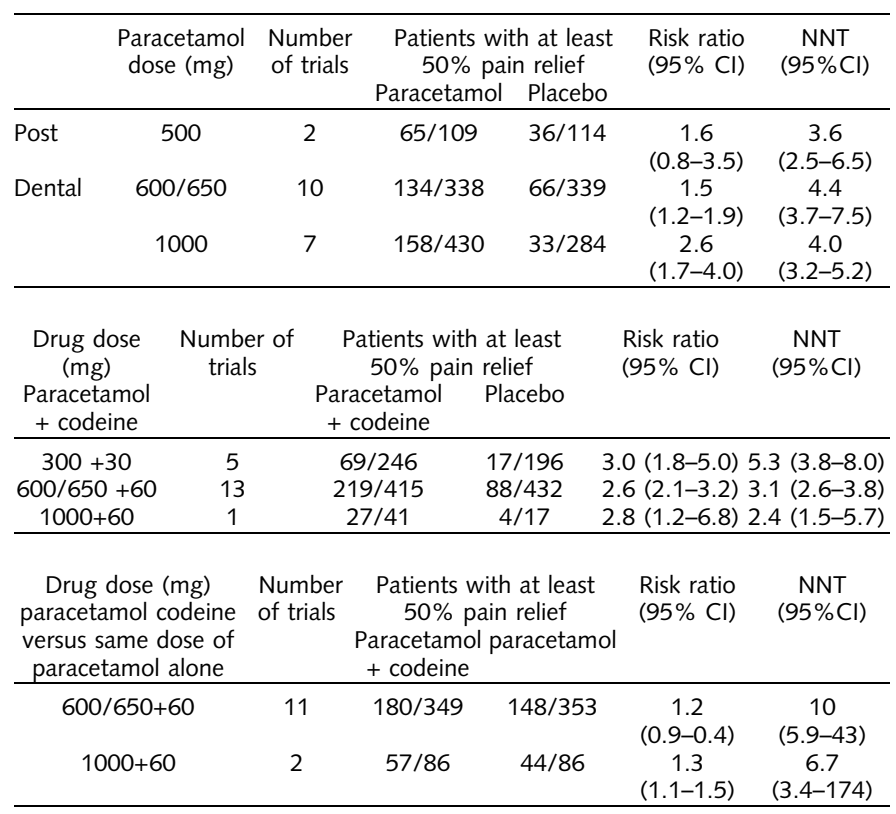

\section{Commentary}

Oral analgesic formulations containing paracetamol in combination with a centrally acting opioid analgesic such as codeine are commonly prescribed for the management of post-operative pain. This paper describes a systematic review of published randomized placebo-controlled clinical trials that evaluated either paracetamol alone or paracetamol plus codeine. Individual studies of paracetamol or codeine, when administered at therapeutic doses, generally confirm that (1) both agents are more effective than placebo and (2) paracetamol is a more effective analgesic than codeine. Individual studies of combination formulations, however, do not consistently demonstrate a statistically significant additive analgesic response. This quantitative systematic review confirms the analgesic efficacy of combined paracetamol plus codeine formulations.

Meta-analyses of analgesic drug efficacy studies require a common outcome measure for measuring pain relief. The outcome measures most frequently used in the third molar extraction model were established by Cooper and Beaver almost 25 years ago ${ }^{1}$. These include pain intensity difference (PID) from baseline at a given time, the summation of pain intensity differences for the duration of the study (SPIDs), pain relief from baseline at a given time (PARs) and the summation of pain relief for the duration of the study (TOTPARs). Other subjective pain measures are based on changes in pain experience quantified using visual analogue scales (VAS). Although these pain measures have been shown to be valid, they tend to have skewed distributions and overestimate efficacy if dropout rates are high.

The authors of this review derive a dichotomous measure called 'numberneeded-to-treat' (NNT), which avoids comparing mean pain relief values. The NNT estimates the number of subjects who would have got at least $50 \%$ pain relief who would not have if they received an alternative agent or placebo. Although the definition of this measure is somewhat cumbersome, it provides a concise summary value with $95 \%$ confidence limits that has been used previously to estimate analgesic efficacy.

The authors have provided a rigorous review of the efficacy of this common analgesic drug combination that supports its use in acute dental post-operative pain management. However, one should appreciate the limitations of their findings. The conclusion is dependent upon using the full therapeutic codeine dose of $60 \mathrm{mg}$; formulations using 15 or 30 mg of codeine may not provide significant improvement in analgesia. Additionally, the combination is likely to induce a higher incidence of nausea and vomiting, an adverse reaction that is likely following outpatient oral surgery. Neither of these clinically important issues has been addressed in this analysis.

1 Cooper SA, Beaver WT. A model to evaluate mild analgesics in oral surgery outpatients. Clin Pharmacol Ther 1976; 20:241-250.

Paul A Moore Professor, Dental Public Health, School of Dental Medicine, University of Pittsburgh, USA 